\title{
Know Your Audience: Comprehension of Health Information Varies by Visual Format
}

Sabrina Mangal, PhD, RN1, Meghan Reading Turchioe, PhD, MPH, RN1, Leslie Park, BS1, Yujie Hai, MS1, Annie C. Myers, MA¹, Lydia Dugdale MD, MAR², Parag Goyal, MD, MSc ${ }^{3}$, Lisa Grossman Liu, PhD4, Ruth M. Masterson Creber, $\mathrm{PhD}, \mathrm{MSc}, \mathrm{RN} 1$

${ }_{1}$ Department of Population Health Sciences, Division of Health Informatics, Weill Cornell Medicine, New York, NY; ${ }^{2}$ Department of Medicine, College of Physicians and Surgeons, Columbia University, New York, NY; ${ }^{3}$ Department of Medicine, Divisions of Cardiology and General Internal Medicine, Weill Cornell Medicine, New York, NY; ${ }^{4}$ Department of Biomedical Informatics, College of Physicians and Surgeons, Columbia University, New York, NY 


\section{Abstract}

Patient-reported outcomes provide key information about a patient's health status and are increasingly being integrated into electronic health records as a means of engaging patients in their own care. However, there is a lack of research on how to effectively return this information in a clear and comprehensible way to a wide audience. We conducted a national survey, testing various formats of health information visualizations, and found that overall comprehension is high across all formats, particularly among line graphs, with variations based on literacy and numeracy. This study reinforces the importance of testing various formats among target populations when returning key health information to patients.

Keywords: Information visualization, patient-reported outcomes, patient communication, visual health information.

\section{INTRODUCTION}

Patient-reported outcomes (PROs) are key indicators of health status that are reported by patients, which include information such as pain, fatigue, quality of life, and functional status [1]. PROs are vitally important to communicate insights about a patient's health that are not objectively available through traditional tests or assessments from clinicians and are useful to inform and facilitate medical decision making and patient communication [2-5]. Federal mandates urge healthcare systems to collect PRO information and share them with patients to encourage transparency and holistic patient care [6-8]. However, a long-standing challenge remains that PRO information is not returned to patients in a way that is comprehensible or helpful for patients with varied levels of health literacy and education [9]. Typically, personal health information is returned to patients using raw numbers and complex medical terminology that are only meaningful to medical professionals, further narrowing the scope of individuals who can benefit from accessing this information.

A potential solution to this issue is to use information visualizations to return $\mathrm{PRO}$ health information to patients. The use of visualizations is an effective approach to communicate health information to patients of varying levels of health literacy and health disparities $[10,11]$. In a study involving hospitalized patients, we tested the comprehension of four PRO visualization formats (text only, number line, line graph, visual analogy), using the results of standardized PRO symptom surveys representing fatigue, depression, and physical function. We found that a visual analogy worked best to communicate our selected constructs of PRO health information [12]. However, this was a small sample of hospitalized patients that does not generalize to the US population. Given that PROs are increasingly being used across healthcare settings and not just among acutely ill patients, it is important to test these visualizations in a larger, national sample. Therefore, the objective of this study is to explore public perspectives on the comprehension, usefulness, and confidence in understanding previously developed PRO information visualizations.

\section{Methods}

A national sample of participants from the United States was recruited from March to April 2021 using Prolific, an academic survey recruitment platform with user verification. Prolific employs a random-sampling algorithm using US Census data to stratify and recruit participants across subgroups that are representative of the population.

We collected demographic information (e.g., age, gender, race, ethnicity, education) and assessed graph literacy, health literacy, and subjective numeracy using validated instruments [13-15]. We provided participants with four visualization conditions (text only, text plus visual analogy, text plus line graph, and text plus number line), representing the symptom "fatigue" in a hypothetical scenario because it is a prevalent symptom among adults.

\subsection{Visualizations}

The visualizations included four formats of health information which were previously developed and tested using user-centered design among patients with heart failure (Figure 1). Each visualization shared a common anatomy with text across the top of all formats that includes a gist interpretation of the graphic (e.g., "Your fatigue got worse"), and included a fatigue score from "two weeks ago" and "today" representing a change over time. The change in score was represented in different formats (text only, visual analogy, line graph, number line). All visualizations utilized a red-yellow-green palette using shades and tints that enable colors to be differentiated for people with color vision deficiency, and colorblindness filters were applied to ensure adequate differentiation.

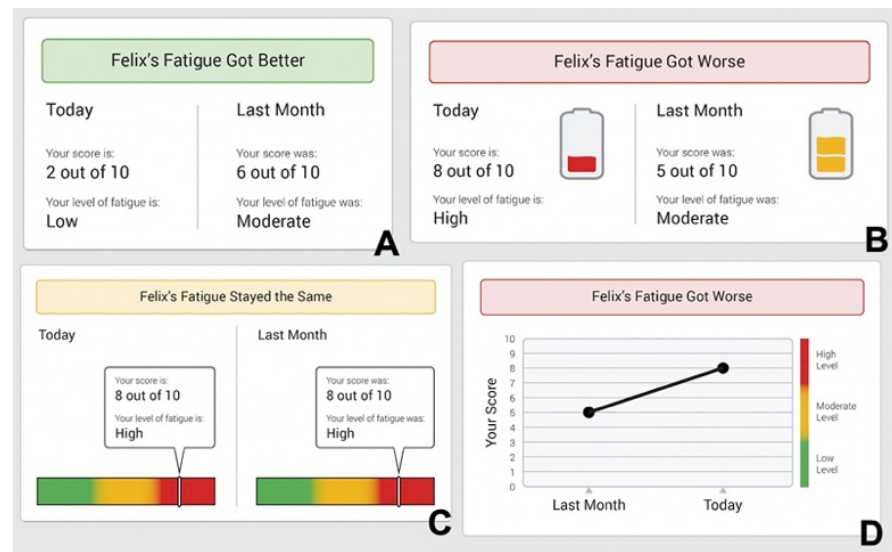

Figure 1: Examples of the four visualization formats displayed to participants. A) Text-only; B) Text plus visual analogy; C) Text plus number line; D) Text plus line graph

\subsection{Procedures}

We used counterbalancing to randomly distribute the order and type of information displayed within the condition sets. We displayed each of the four visualization formats one at a time, prefaced by an introductory phrase, "Felix received the following health summary about his fatigue". Following each visualization, by three comprehension questions evaluating gist and verbatim comprehension, a method that is aligned with previous research [16]: What was Felix's fatigue score last month? (Q1, verbatim); What is Felix's fatigue level today? (Q2, verbatim); and How is Felix's fatigue today compared to last month? (Q3, gist). After the comprehension questions, we asked participants to indicate on a scale of 1 (Not useful at all) to 10 (Extremely useful): How useful this health summary would be for understanding your own health information?, and on a 5-point scale from "Not confident at all" to "Extremely confident": How confident do you feel that you could understand this health summary if researchers returned it to you?

\subsection{Analysis}

We conducted descriptive and inferential analyses using $\mathrm{R}$ version 4.0.5. We used descriptive statistics to summarize demographics, overall comprehension, comprehension based on format, and confidence and usefulness of the visualizations. We conducted bivariate analyses using logistic regression to determine whether 
demographic characteristics were independently associated with comprehension. The comprehension outcome variable was defined as high ( 3 out of 3 comprehension questions correct) or low ( 2 or fewer comprehension questions correct) across all formats and for each specific format. We used the Test of Equal or Given Proportions to determine whether comprehension scores were significantly different from one another across formats. The confidence outcome variable was categorized as: highly confident (4 or 5 out of 5), moderately confident ( 3 out of 5), and not confident ( 1 or 2 out of 5 . The usefulness variable was categorized as: highly useful ( 7 to 10 out of 10$)$, moderately useful (5 or 6 out of 10), or not useful (1 to 4 out of 10).

\section{Results}

Demographic characteristics are summarized in Supplementary Table 1. Overall, participants $(n=502)$ had an average age of 46.6 ( \pm 16.0 ) years and were $51 \%$ female, $74 \%$ White, and $7 \%$ Hispanic or Latino. Furthermore, $66 \%$ attended some college or had a bachelor's degree, $33 \%$ had low subjective numeracy scores, $28 \%$ had limited/marginal health literacy and $23 \%$ had poor/very poor graph literacy scores.

\subsection{Comprehension}

Most participants had high comprehension of the text-only (89\%), number line $(89 \%)$, with visual analogy $(92 \%)$, and line graph $(94 \%)$ being the highest scoring formats $(\mathrm{p}=0.01)$. A large proportion of participants answered correctly to verbatim comprehension questions 1 (text only: $96 \%$ correct, number line: 95\% correct, visual analogy: 97\% correct, line graph: $98 \%$ ) and 2 (text only: $98 \%$ correct, number line: $97 \%$ correct, visual analogy: 97\% correct, line graph: 98\%). Responses to the gist comprehension question were also high (text only: $92 \%$ correct, number line: $93 \%$ correct, visual analogy: $94 \%$ correct, line graph: $96 \%)$.

\subsection{Usefulness and Confidence in Understanding}

Most participants rated each format as "moderately useful" in understanding health information: text only (70\%), line graphs (70\%), number lines (72\%), and visual analogies (73\%). Additionally, most respondents responded that they were "highly confident" that they understood each format: line graph $(65 \%)$, text only (70\%), number line (70\%), and visual analogy (71\%).

\subsection{Comprehension Analyses}

All associations identified between comprehension and formats are detailed in Supplementary Table 1. For comprehension across all formats combined, low subjective numeracy, medium subjective numeracy, and poor/very poor graph literacy were independently associated with lower overall comprehension. For text-only comprehension, low subjective numeracy, poor/very poor graph literacy, and no confidence in understanding the format were independently associated with lower comprehension. For visual analogy comprehension, poor/very poor graph literacy, and no confidence in understanding the format were independently associated with lower comprehension. For number line comprehension, problems with seeing, hearing, or mobility, low subjective numeracy, poor/very poor graph literacy, moderate confidence in understanding the format, and no confidence in understanding the format were independently associated with lower comprehension. Lastly, for line graphs, Asian race was independently associated with lower comprehension of the line graphs. Figure 2 details the odds ratios and confidence intervals of associations between comprehension and low subjective numeracy and poor/very poor graph literacy, across each format.

\section{Low Subjective Numeracy vs. Comprehension}

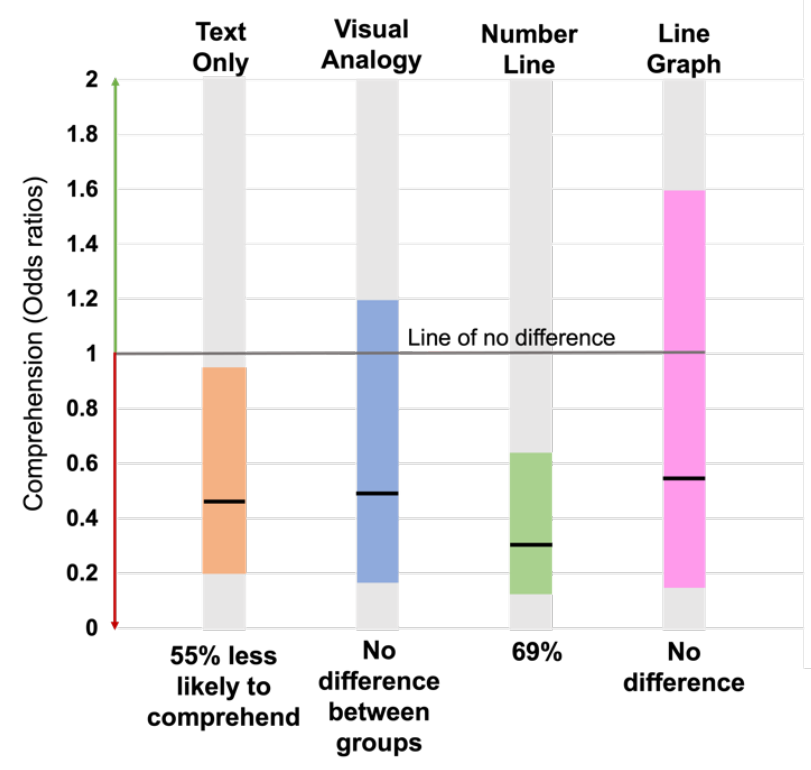

\section{Poor/Very Poor Graph Literacy vs. Comprehension}

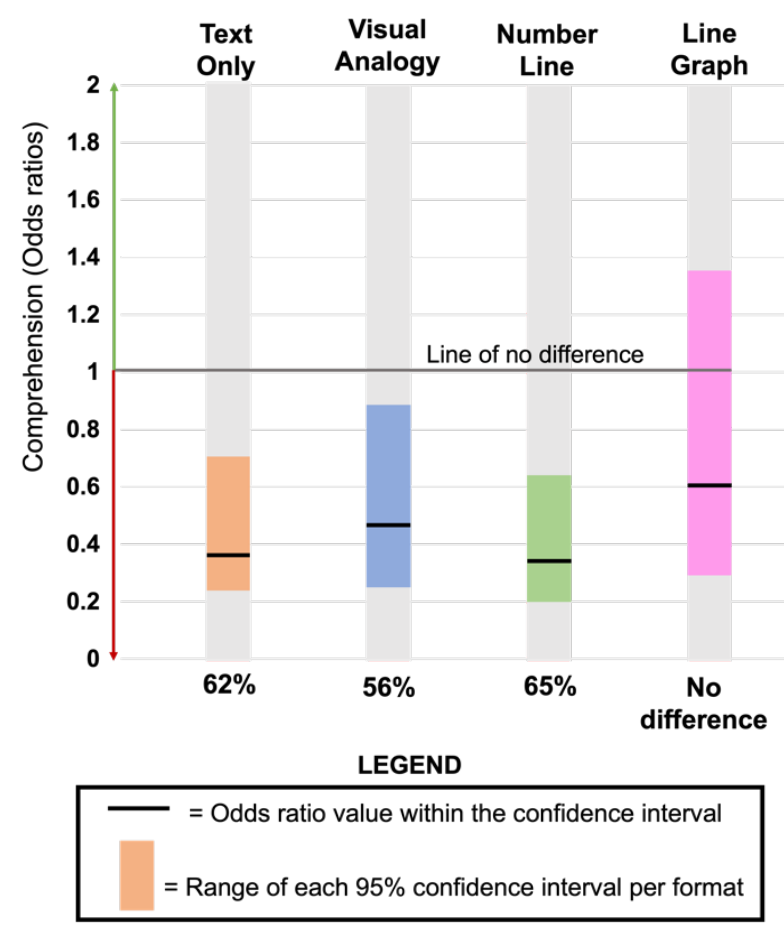

Figure 2: Associations between individuals with low subjective numeracy (compared to high subjective numeracy) and comprehension, and poor/very poor graph literacy (compared to good/fair graph literacy) and comprehension of a patient-reported outcome symptom report across 4 visualization formats. 


\section{Discussion}

In this study of over 500 participants from across the US, we report high comprehension across all visual formats with variations across comprehension by level of subjective numeracy and graph literacy. Generally, participants found the formats moderately useful. Additionally, participants had high comprehension levels and high confidence in understanding the formats if they were returned to them. Overall, these study findings support the return of PRO information summaries in formats that provide context and support interpretability.

We found that line graphs were the most highly comprehended format, with no differences in comprehension based on graph literacy, health literacy, or subjective numeracy. Notably, the line graph was the only format that did not include text interpretations of the verbatim questions, but rather required participants to interpret the line graph to extrapolate the verbatim answers. This suggests that our participants had a moderate baseline level of familiarity with line graphs. Prior studies align with the findings of this study to support the use of line graphs [17] but have compared objective comprehension only among line graphs or compared subjective ease of interpretation among several formats including line graphs [18]. We confirmed these findings by comparing objective comprehension across several formats. Our study findings are inconsistent with one prior study showing the visual analogy format was the most comprehended format for returning PRO information and line graphs were the least comprehended formats [12]. However, there are important differences in the two samples; the prior study was conducted with an acutely ill, older population, whereas our sample included a younger population with higher literacy and numeracy levels. Variations between these findings reiterate the importance of knowing your audience to return health information in a way that is most comprehensible to the target population.

We also found that low subjective numeracy scores and/or poor/very poor graph literacy scores were associated with lower comprehension of text-only, visual analogy, and number line formats. Aligned with existing research, it is important to measure and report literacy and numeracy scores when testing various formats for comprehension, which can help researchers identify elements of visualizations that can be improved upon for vulnerable populations [19-21]. Most participants found all visual formats to be "moderately useful" for understanding their health information. Future work could go beyond comprehension to determine the best method for supporting desired responses to visualizations, such as self-management. Prior research highlights that a vast majority of patients $(78 \%)$ would show their health information summary to a clinician, but only $10 \%$ would take action to self-manage their health condition [12]. Future research should further explore how varying formats of information can impact actionability and behavioral intent for self-management and communication.

There were several limitations to this study. First, we used an online recruitment platform for participants, which limits our sample to individuals who are familiar with using technology. Also, our sample was highly educated and generally had higher levels of graph literacy, health literacy, and subjective numeracy compared to the general US public. Lastly, we did not measure actionability or behavioral intention after presenting visualizations, which limits the scope of our conclusions.

\section{Conclusion}

Overall, we found that participants had generally high levels of comprehension and subjective usefulness across all formats of PRO visualizations, with variations by graph literacy and subjective numeracy. Findings emphasize the need to examine the needs and preferences of the target audience when considering the return of health information.

\section{ACKNOWLEDGEMENTS}

This work is funded by the National Institute of Nursing Research (R00NR016275; R00NR016275-05S1).

\section{References}

[1] Weldring T, Smith SMS. Patient-Reported Outcomes (PROs) and Patient-Reported Outcome Measures (PROMs). Health Serv Insights, 6:61-68, 2013

[2] Au HJ, Ringash J, Brundage M, Palmer M, Richardson H, Meyer RM. Added value of health-related quality of life measurement in cancer clinical trials: The experience of the NCIC CTG. Expert Rev Pharmacoecon Outcomes Res, 2:119-128, 2010.

[3] Detmar SB, Muller MJ, Schornagel JH, Wever LDV, Aaronson NK. Health-related quality-of-life assessments and patient-physician communication: a randomized controlled trial. JAMA. 288(23):30273034, 2002.

[4] Greenhalgh J. The applications of PROs in clinical practice: What are they, do they work, and why? Qual Life Res. 18(1):115-123, 2009.

[5] Berry DL et al., Enhancing patient-provider communication with the electronic self-report assessment for cancer: A randomized trial. J Clin Oncol. 29(8):1029-1035, 2011.

[6] Gensheimer SG et al., Oh, the places we'll go: Patient-reported outcomes and electronic health records. Patient-Centered Outcomes Res. 11(6):591-598, 2018.

[7] Wagle NW. Implementing patient-reported outcome measures. NEJM Catal., October 2017. (accessed 2021).

[8] Jensen RE, Snyder CF, Basch E, Frank L, Wu AW. All together now: Findings from a PCORI workshop to align patient-reported outcomes in the electronic health record. J Comp Eff Res. 5(6):561-567, 2016.

[9] Irizarry T, DeVito Dabbs A, Curran CR. Patient portals and patient engagement: A state of the science review. J Med Internet Res. 17(6), 2015.

[10] Arcia A, George M, Lor M, Mangal S, Bruzzese JM. Design and comprehension testing of tailored asthma control infographics for adults with persistent asthma. Appl Clin Inform . 10(4):643-654, 2019.

[11] Arcia A et al., Sometimes more is more: Iterative participatory design of infographics for engagement of community members with varying levels of health literacy. J Am Med Inform Assoc. 23(1):174-183, 2016.

[12] Reading Turchioe M, Grossman LV, Myers AC, Baik D, Goyal P, Masterson Creber RM. Visual analogies, not graphs, increase patients' comprehension of changes in their health status. J Am Med Inform Assoc. 27(5):677-689, 2020

[13] Chew LD et al., Validation of screening questions for limited health literacy in a large VA outpatient population. J Gen Intern Med. 23(5):561-566, 2008.

[14] Okan Y, Janssen E, Galesic M, Waters EA. Using the short graph literacy scale to predict precursors of health behavior change. Int J Soc Med Decis Mak. 39(3):183-195, 2019.

[15] McNaughton CD, Cavanaugh KL, Kripalani S, Rothman RL, Wallston KA. Validation of a short, 3-item version of the Subjective Numeracy Scale. J Soc Med Decis Mak. 35(8):932-936, 2015.

[16] Arcia A, Grossman LV, George M, Turchioe Reading M, Mangal S, Masterson Creber RM. Modifications to the ISO 9186 method for testing comprehension of visualizations: Successes and lessons learned. In: 2019 IEEE Workshop on Visual Analytics in Healthcare (VAHC); 41-47, 2019

[17] Snyder CF, Smith KC, Bantug ET, Tolbert EE, Blackford AL, Brundage MD. What do these scores mean? Presenting patientreported outcomes data to patients and clinicians to improve interpretability. Cancer. 123(10):1848-1859, 2017. 
[18] Brundage MD, Smith KC, Little EA, Bantug ET, Snyder CF. Communicating patient-reported outcome scores using graphic formats: Results from a mixed-methods evaluation. Int J Qual Life Asp Treat Care Rehabil. 24(10):2457-2472, 2015.

[19] Turchioe Reading $M$ et al., A systematic review of patient-facing visualizations of personal health data. Appl Clin Inform. 10(4):751$770,2019$.

[20] Zikmund-Fisher BJ et al., Graphics help patients distinguish between urgent and non-urgent deviations in laboratory test results. J Am Med Inform Assoc. 24(3):520-528, 2017.

[21] Martinez W, Threatt AL, Rosenbloom ST, Wallston KA, Hickson GB, Elasy TA. A patient-facing diabetes dashboard embedded in a patient web portal: Design sprint and usability testing. JMIR Hum Factors. 5(3):e26, 2018. 
Supplementary Table 1

Summary of demographic characteristics and associations between sociodemographic factors and comprehension overall and by format

\begin{tabular}{|c|c|c|c|c|c|c|}
\hline \multirow{2}{*}{$\begin{array}{l}\text { Demographic } \\
\text { Characteristics }\end{array}$} & \multirow{2}{*}{$\begin{array}{l}\text { Total } \\
\text { n }(\%)\end{array}$} & \multirow{2}{*}{$\begin{array}{c}\text { Overall } \\
\text { Comprehension } \\
(\mathrm{OR}[\mathrm{CI}])\end{array}$} & \multicolumn{4}{|c|}{ Format Delivered } \\
\hline & & & $\begin{array}{l}\text { Text Only } \\
(\mathrm{OR}[\mathrm{CI}])\end{array}$ & $\begin{array}{l}\text { Visual Analogy } \\
\text { (OR [CI]) }\end{array}$ & $\begin{array}{c}\text { Number Line } \\
\text { (OR }[\mathrm{CI}])\end{array}$ & $\begin{array}{c}\text { Line Graph } \\
(\mathrm{OR}[\mathrm{CI}])\end{array}$ \\
\hline Age (mean, SD) & $46.6(16)$ & $1.01[0.99-1.02]$ & $1.00[0.99-1.02]$ & 0.99 [0.97-1.01] & $1.00[0.98-1.02]$ & $1.02[0.99-1.04]$ \\
\hline \multicolumn{7}{|l|}{ Gender } \\
\hline Female & $254(51)$ & Ref & & & & \\
\hline Male & $243(48)$ & $0.97[0.63-1.48]$ & $0.78[0.45-1.36]$ & $0.90[0.47-1.74]$ & $0.84[0.48-1.47]$ & $0.78[0.37-1.61]$ \\
\hline Other & $5(1.0)$ & --- & --- & --- & --- & --- \\
\hline Asian & $36(7.2)$ & 0.71 [0.34- 1.59] & $1.05[0.39-3.66]$ & $1.50[0.43-9.52]$ & $1.48[0.50-6.35]$ & $0.28[0.10-0.90]^{*}$ \\
\hline Black or African & $65(13)$ & $0.77[0.42-1.44]$ & $1.09[0.49-2.75]$ & $0.73[0.32-1.88]$ & $0.96[0.45-2.30]$ & $0.44[0.17-1.28]$ \\
\hline \multicolumn{7}{|l|}{ American } \\
\hline $\begin{array}{l}\quad \text { Native } \\
\text { American/Alaska } \\
\text { Native }\end{array}$ & $5(1)$ & --- & --- & --- & --- & --- \\
\hline \multicolumn{7}{|l|}{ Latino Origin } \\
\hline \multicolumn{7}{|l|}{ Education } \\
\hline $\begin{array}{l}\text { Associate or } \\
\text { bachelors }\end{array}$ & $223(45)$ & $1.43[.68-2.88]$ & $1.97[0.77-4.63]$ & $1.39[0.38,4.09]$ & $1.39[0.52-3.28]$ & $2.21[0.58-7.13]$ \\
\hline Some college & $96(19)$ & $1.41[0.64-3.02]$ & $1.19[0.45-2.87]$ & $0.85[0.23-2.60]$ & $1.47[0.52-3.85]$ & $1.33[0.34-4.44]$ \\
\hline Graduate & $110(22)$ & $1.12[0.51-2.39]$ & $2.05[0.73-5.58]$ & $0.76[0.20-2.32]$ & $1.43[0.50-3.82]$ & $0.93[0.24-2.95]$ \\
\hline \multicolumn{7}{|l|}{ Financial resources } \\
\hline Enough & $297(59)$ & Ref & & & & \\
\hline Not enough & $126(25)$ & $0.94[0.57-1.56]$ & $0.83[0.44-1.61]$ & 0.87 [0.43-1.86] & $0.86[0.47-1.63]$ & $0.90[0.37-2.42]$ \\
\hline \multicolumn{7}{|l|}{ enough } \\
\hline \multicolumn{7}{|l|}{ Disability status } \\
\hline Adequate & $363(72)$ & Ref & & & & \\
\hline Limited & $139(28)$ & $1.03[0.65-1.68]$ & $0.81[0.45-1.50]$ & $1.25[0.62-2.74]$ & $0.81[0.45-1.50]$ & $0.93[0.43-2.18]$ \\
\hline \multicolumn{7}{|l|}{ Subjective Numeracy } \\
\hline High & $129(26)$ & Ref & & & & \\
\hline Low & $165(33)$ & $0.43[0.23-0.78]^{*}$ & $0.45[0.20-0.94]^{*}$ & $0.50[0.19-1.20]$ & $0.31[0.12-0.70]^{*}$ & $0.56[0.17-1.60]$ \\
\hline Medium & $208(41)$ & $0.50[0.27-0.89]^{*}$ & $0.75[0.33-1.61]$ & $0.61[0.23-1.44]$ & $0.44[0.17-1.00]$ & $0.52[0.17-1.38]$ \\
\hline \multicolumn{7}{|l|}{ Graph Literacy } \\
\hline Good/Fair & $388(77)$ & Ref & & & & \\
\hline Poor/Very Poor & $114(23)$ & $0.44[0.28-0.69]^{*}$ & $0.38[0.21-0.68]^{*}$ & $0.44[0.23-0.87]^{*}$ & $0.35[0.20-0.62]^{*}$ & $0.60[0.28-1.36]$ \\
\hline \multicolumn{7}{|l|}{ Confidence } \\
\hline High & N/A & N/A & Ref & & & \\
\hline Moderate & N/A & N/A & $0.55[0.28-1.15]$ & $1.25[0.53-3.44]$ & $0.36[0.19-0.71]^{*}$ & $0.69[0.30-1.75]$ \\
\hline Not confident & N/A & N/A & $0.26[0.13-0.53]^{*}$ & $0.39[0.17-0.92]^{*}$ & $0.23[0.11-0.49]^{*}$ & $0.37[0.15-1.01]$ \\
\hline \multicolumn{7}{|l|}{ Usefulness } \\
\hline High & N/A & N/A & Ref & & & \\
\hline Moderate & N/A & N/A & $1.36[0.67-2.62]$ & $1.93[0.85-4.08]$ & $1.79[0.87-3.51]$ & $0.60[0.17-1.62]$ \\
\hline Not useful & N/A & N/A & $0.87[0.34-2.34]$ & $1.23[0.43-3.81]$ & $0.74[0.30-1.82]$ & $0.48[0.11-2.13]$ \\
\hline
\end{tabular}

\footnotetext{
* indicates statistical significance; Ref $=$ Reference group; N/A = Not applicable; $---=$ Model did not converge due to small sample size; HS = High school; Disability = Problems with seeing, hearing, and/or mobility; Confidence = Confidence in understanding the format; Usefulness = Usefulness of the format to understand health information.
} 